(1)

2

3

7

8

\title{
Development and validation of a computational model for steak double-sided pan cooking
}

\author{
J. Moya $^{\mathrm{a}}$, S. Lorente-Bailo ${ }^{\mathrm{b}}$, M. L. Salvador ${ }^{\mathrm{b}}$, A. Ferrer-Mairal $^{\mathrm{b}}$, M. A. \\ Martínez $^{\text {a,c }}$, B. Calvo ${ }^{\mathrm{a}, \mathrm{c}}$, J. Grasa ${ }^{\mathrm{a}, \mathrm{c}}$ \\ ${ }^{a}$ Aragón Institute of Engineering Research (i3A), Universidad de Zaragoza, Spain. \\ ${ }^{b}$ Plant Foods Research Group, Instituto Agroalimentario de Aragon IA2, Universidad de \\ Zaragoza-CITA, Miguel Servet 177, 50013 Zaragoza, Spain \\ ${ }^{c}$ Centro de Investigación Biomédica en Red en Bioingeniería, Biomateriales y \\ Nanomedicina (CIBER-BBN), Spain.
}

10

The objective of this study was to develop and validate a numerical model to adequately simulate the double-sided pan cooking of beef in a domestic environment. The proposed model takes into account the heat flow from the pan to the meat and the moisture transfer, simultaneously with the meat deformation. The model considers the swelling pressure gradient caused by the shrinkage of the meat fibers and connective tissue due to the denaturation of proteins and the loss of the water holding capacity during cooking. The model results were successfully verified with experimental data of the central temperature and weight loss recorded during cooking for three degrees of doneness. The measured experimental temperatures at the center of the meat were $30 \pm 3{ }^{\circ} \mathrm{C}$ (very rare), $44 \pm 3{ }^{\circ} \mathrm{C}$ (rare) and $57 \pm 2{ }^{\circ} \mathrm{C}$ (done) for a $19 \mathrm{~mm}$ steak thickness. Meanwhile, their water losses were $4 \pm 2 \%$, $8 \pm 1 \%$ and $11 \pm 2 \%$, respectively. The root mean squared errors of the model predictions were $2.16{ }^{\circ} \mathrm{C}$ (very rare), $3.56{ }^{\circ} \mathrm{C}$ (rare) and $4.57^{\circ} \mathrm{C}$ (done) for the central temperature and $1.48 \%, 2.08 \%$ and $2.40 \%$, respectively for 
the water loss. The model also correctly predicts cooking times for steaks of different thicknesses, taking weight loss as a reference to set this time. The proposed model is postulated as a useful cooking assistance tool to estimate the optimal cooking time according to consumer preferences. Keywords: Cooking, food model, beef meat, shrinkage, finite elements

\section{Introduction}

There is increasing interest in developing accurate numerical models of meat cooking processes with the aim of achieving a high degree of knowledge and control of the complex heat and mass transfer phenomena involved. Knowledge of meat behavior during cooking is very important for optimizing and controlling the final quality of the product. The physical phenomena that underlie the meat cooking process can basically be considered by the coupling of heat and moisture transfer in a deforming porous medium (Datta, 2007). The state of the art models differ in their degrees of approximation and therefore in their complexity. Some exclusively consider conductive heat transfer and the diffusive transport of matter. Other models incorporate the convective heat transport by the liquid moisture flow and also describe the moisture transport by the Flory-Rehner theory (van der Sman, 2015; Feyissa et al., 2013; Ahmad et al., 2015; Nelson et al., 2020). However, little information has been provided on meat deformation during cooking as a solid mechanics problem, with some exceptions such as the research conducted by Dhall and Datta (2011), Feyissa et al. (2013) and Blikra et al. (2019). When meat is heated, water migrates through the surface either in the form of liquid or in the form of vapor whilst the temperature and water content inside the 
meat vary in space and time at the same time that the meat volume changes. During cooking, meat proteins denature and cause structural changes, such as the shrinkage of muscle fibers and connective tissue, and the formation of larger pores in parts closer to the surface, being smaller in parts closer to the center (Feyissa et al., 2013). These changes in porosity lead to an increase in water permeability in the outer parts of the meat and consequently in the water transport. Not considering this shrinkage could lead to errors in the estimation of the weight loss of the meat (Datta, 2016). These structural changes also decrease the water holding capacity of the meat. The mechanical force exerted by the contracting protein network on the interstitial fluid, denoted swelling pressure, leads to the expulsion of the moisture from the meat (Tornberg, 2005). In water loss, swelling pressure is a more important mechanism than surface evaporation, accounting for up to $80 \%$ of the water losses during double-sided pan cooking of beef burgers (Tornberg, 2013). In these cases, Darcy's law is used to associate the hydraulic pressure with the moisture transport (Rabeler and Feyissa, 2018).

Many recent meat cooking models have focused on baking or frying (Ahmad et al., 2015; Bansal et al., 2015; Feyissa et al., 2013; Isleroglu and Kaymak-Ertekin, 2016; Kondjoyan et al., 2013; Rabeler and Feyissa, 2018; van der Sman, 2007; van Koerten et al., 2017) and only a few on pan cooking (Dhall and Datta, 2011; Eberth et al., 2012; Rocca-Poliméni et al., 2019) despite being a very common cooking method in the domestic environment. This may be due to the added complexity of tracking the heat transfer phenomena between the pan surface and the meat. The real contact surface between the meat and the pan is smaller than what is apparent because it can 
be considered as interspersed contact spots between gaps (Rocca-Poliméni et al., 2019). Another challenge of pan cooking modeling is the need to turn the meat over. Previous studies have been limited to the analysis of onesided pan cooking of the meat, when typically the meat is turned several times during cooking (Myhrvold, 2017).

The main objective of this work was to define and validate a computational model that, in addition to describing the coupled transfer of heat and humidity during the domestic pan cooking of beef meat, takes into account: i) the deformation of the meat as a solid mechanical problem, ii) includes the turn over process, and iii) addresses some key aspects of pan cooking as the contact heating interface between the pan and the meat. In order to validate the model, an experimental protocol was developed to gather information during meat cooking and to obtain some of the beef meat properties that the model needs as inputs. The computational results were verified by comparing the temperature and the weight loss evolution of beef with the results obtained by experimentation. Another objective of this study was to check the adaptability of the proposed model for use as assistance to cooking in a domestic environment. For this reason, the cooking times predicted by the model for different thicknesses of the meat piece were experimentally verified.

\section{Mathematical model}

The pan-cooking of beef can be described as a flow and transport problem in a deforming solid matrix during thermal processing. In this study, the mathematical model that describes these phenomena was implemented in the software COMSOL Multiphysics 5.2a while making the following as- 
sumptions: (1) Meat was considered as a continuum biphasic (liquid-solid) porous material. For simplicity, the structure of the meat was assumed to be homogeneous, since in this case, the majority of the meat does not reach very high temperatures so that the existence of larger pores on the surface can be neglected. (2) Due to the rubbery nature of the meat, it was addressed as a hyper-elastic material in which the total volume change was equal to the volume of moisture loss and consequently the solid matrix remained saturated. (3) The temperature was assumed to be the same for the two phases. (4) The moisture flow due to the pressure gradient caused by the shrinking connective tissue on the aqueous solution in the extracellular void followed Darcy's law, (5) Water evaporates on the surface of the meat which is in contact with the pan.

It is convenient, at this point, to introduce the volume fraction $\phi_{\alpha}$ for each phase:

$$
\phi_{\alpha}=\lim _{V \rightarrow 0} \frac{V_{\alpha}}{V}, \quad \alpha=s, f
$$

where $V_{\alpha}$ is the volume occupied by the $\alpha$ phase and $V=V_{s}+V_{f}$ is the total volume. The volume fractions $\phi_{\alpha}$ in (1) satisfy the volume fraction condition $\phi_{s}+\phi_{f}=1$. The density of the solid and fluid phase is related to its true (or, intrinsic) density $\bar{\rho}_{\alpha}$ as follows:

$$
\rho_{\alpha}=\bar{\rho}_{\alpha} \phi_{\alpha}, \quad \alpha=s, f
$$

To describe the kinematics or motion of the biphasic media, let $\boldsymbol{x}=$ $\boldsymbol{\chi}(\boldsymbol{X}, t): \Omega_{0} \times \mathbb{R} \rightarrow \mathbb{R}^{3}$ denotes the motion mapping and let $\boldsymbol{F}$ be the associated deformation gradient. Here $\boldsymbol{X}$ and $\boldsymbol{x}$ define the respective positions of 
a particle in the reference $\Omega_{0}$ and current $\Omega$ configurations such that $\boldsymbol{F}=\frac{d \boldsymbol{x}}{d \boldsymbol{X}}$ represents a measure of the deformation. Further, let $J \equiv \operatorname{det} \boldsymbol{F}$ be the Jacobian of the motion that provides the ratio between the volume in the present configuration and the volume in the reference configuration.. To properly define volumetric and deviatoric responses in the nonlinear range, we introduce the following kinematic decomposition (Flory, 1961):

$$
\begin{array}{ll}
\boldsymbol{F}=J^{\frac{1}{3}} \overline{\boldsymbol{F}}, & \overline{\boldsymbol{F}}=J^{-\frac{1}{3}} \boldsymbol{F} \\
\boldsymbol{C}=\boldsymbol{F}^{T} \boldsymbol{F}, & \overline{\boldsymbol{C}}=J^{-\frac{2}{3}} \boldsymbol{C}=\overline{\boldsymbol{F}}^{T} \overline{\boldsymbol{F}}
\end{array}
$$

where $J^{\frac{1}{3}}$ and $\overline{\boldsymbol{F}}$ represent the volumetric and distortional components, respectively. $\overline{\boldsymbol{F}}$ and $\overline{\boldsymbol{C}}$ are denoted as the modified deformation gradient and the modified right Cauchy-Green tensors.

\section{[Figure 1 about here.]}

Assuming that the deformation of the meat due to temperature effects is small and therefore can be neglected, the moisture loss and deformation of the meat can be modelled as two fictitious processes (Vujosevic and Lubarda, 2002; Dhall and Datta, 2011) as can be observed in Fig. 1. Using a multiplicative decomposition of the deformation gradient tensor $\boldsymbol{F}$ :

$$
\boldsymbol{F}=\boldsymbol{F}_{f} \boldsymbol{F}_{s}
$$

The current deformation gradient is the product of the deformation associated with water volume changes $\left(\boldsymbol{F}_{f}\right)$ and the elastic deformation of the solid phase $\left(\boldsymbol{F}_{s}\right)$. The volumetric part of the elastic tensor could be written 
as a function of the deviatoric part taking the Jacobian as $\boldsymbol{F}_{s}=J_{s}^{1 / 3} \overline{\boldsymbol{F}}_{s}$, whereas the water loss implies only a pure volumetric process $\boldsymbol{F}_{f}=J_{f}^{1 / 3} \boldsymbol{I}$, being $\boldsymbol{I}$ the identity matrix. Strain measures can be obtained for both solid and fluid phases in the same way as in Eq. (4). The modified right CauchyGreen tensor for the solid phase $\overline{\boldsymbol{C}}_{s}=\overline{\boldsymbol{F}}_{s}^{T} \overline{\boldsymbol{F}}_{s}$ will be used later for constitutive modelling.

In the absence of body forces and accelerations, conservation of the linear momentum for the bulk material results in the quasi-static equilibrium equation $\nabla \boldsymbol{\sigma}=0$, with $\boldsymbol{\sigma}$ being the total Cauchy stress. Conservation of the angular momentum yields the symmetry of this stress tensor that can be expressed as the sum of the partial solid stress and the partial fluid stress:

$$
\boldsymbol{\sigma}=\hat{\boldsymbol{\sigma}}_{s}+\hat{\boldsymbol{\sigma}}_{f}
$$

where

$$
\hat{\boldsymbol{\sigma}}_{s}=\phi_{s} \boldsymbol{\sigma}_{s} \quad \text { and } \quad \hat{\boldsymbol{\sigma}}_{f}=\phi_{f} \boldsymbol{\sigma}_{f}=-\phi_{f} p_{f} \boldsymbol{I}
$$

$p_{f}$ being the pore fluid pressure.

In this study, the behaviour of meat during the cooking process has, due to its rubbery nature, been approximated using the isotropic Neo-Hookean material model. This model, for the best numerical performance, takes this particular quasi-incompressible form of the strain energy function:

$$
\Psi_{s}\left(\boldsymbol{C}_{s}\right)=\Psi_{s}\left(J_{s}, \overline{\boldsymbol{C}}_{s}\right)=\frac{K}{2}\left(J_{s}-1\right)^{2}+\frac{G^{\prime}}{2}\left(\bar{I}_{1}-3\right)
$$

where $K$ and $G^{\prime}$ are the bulk and the shear elastic modulus and $\bar{I}_{1}=\operatorname{tr} \overline{\boldsymbol{C}}_{s}$ is the first invariant of the modified (deviatoric) right Cauchy-Green tensor. 
Applying the entropy inequality, the second Piola-Kirchhoff stress tensor is obtained as the derivative of the strain energy in a non-dissipative process:

$$
\boldsymbol{S}_{s}=2 \frac{\partial \Psi\left(J_{s}, \overline{\boldsymbol{C}}_{s}\right)}{\partial \boldsymbol{C}_{s}}=\boldsymbol{S}_{s, v o l}+\overline{\boldsymbol{S}}_{s}=J_{s} p_{s} \boldsymbol{C}_{\boldsymbol{s}}^{-1}+J_{s}^{-\frac{2}{3}}\left(\mathbb{I}-\frac{1}{3} \boldsymbol{C}_{s}^{-1} \otimes \boldsymbol{C}_{s}\right): \widetilde{\boldsymbol{S}}_{s}
$$

where $\boldsymbol{S}_{s, \text { vol }}$ and $\overline{\boldsymbol{S}}_{s}$ are the volumetric and deviatoric parts of the second Piola-Kirchhoff stress tensor, $p_{s}$ is the hydrostatic pressure and $\widetilde{\boldsymbol{S}}_{s}$ the modified second Piola-Kirchhoff stress tensor:

$$
p_{s}=\frac{d \Psi_{s, v o l}\left(J_{s}\right)}{d J_{s}} \quad \widetilde{\boldsymbol{S}}_{s}=2 \frac{\partial \bar{\Psi}_{s}\left(\overline{\boldsymbol{C}}_{s}\right)}{\partial \overline{\boldsymbol{C}}_{s}}
$$

For the Neo-Hookean model the explicit expression for the second PiolaKirchhoff stress tensor, as a function of the defined invariant, $\bar{I}_{1}$, is:

$$
\boldsymbol{S}_{s}=J_{s} p_{s} \boldsymbol{C}_{s}^{-1}+2\left[\frac{\partial \bar{\Psi}_{s}}{\partial \bar{I}_{1}} \boldsymbol{I}-\frac{1}{3}\left(\frac{\partial \bar{\Psi}_{s}}{\partial \bar{I}_{1}} \bar{I}_{1}\right) \boldsymbol{C}_{s}^{-1}\right]
$$

The Cauchy stress tensor $\boldsymbol{\sigma}_{s}$ is $1 / J_{s}$ times the push-forward of $\boldsymbol{S}_{s}\left(\boldsymbol{\sigma}_{s}=\right.$ $\left.J_{s}^{-1} \boldsymbol{\chi}_{*}\left(\boldsymbol{S}_{s}\right)\right)$ so, from (9), we obtain

$$
\boldsymbol{\sigma}_{s}=p_{s} \boldsymbol{I}+\frac{2}{J_{s}} \operatorname{dev}\left[\overline{\boldsymbol{F}}_{s} \frac{\partial \bar{\Psi}_{s}\left(\overline{\boldsymbol{C}}_{s}\right)}{\partial \overline{\boldsymbol{C}}_{s}} \overline{\boldsymbol{F}}_{s}^{T}\right]=p_{s} \boldsymbol{I}+\frac{2}{J_{s}}\left(\frac{\partial \bar{\Psi}_{s}}{\partial \bar{I}_{1}} \overline{\boldsymbol{b}}_{s}-\frac{1}{3} \frac{\partial \bar{\Psi}_{s}}{\partial \bar{I}_{1}} \bar{I}_{1} \boldsymbol{I}\right)
$$

with $\boldsymbol{I}$ being the second-order identity tensor and dev the deviator operator in the spatial description and $\overline{\boldsymbol{b}}_{s}=\overline{\boldsymbol{F}}_{s}^{T} \overline{\boldsymbol{F}}_{s}$ the modified left Cauchy-Green tensor.

The product density in the deformed configuration $\rho=\rho(t)$ is:

$$
\rho=\phi_{s} \bar{\rho}_{s}+\phi_{f} \bar{\rho}_{f}=\rho_{s}+\rho_{f}
$$


The mass balance for both phases becomes:

$$
\begin{gathered}
\frac{\partial\left(\phi_{s} \bar{\rho}_{s}\right)}{\partial t}+\nabla\left(\phi_{s} \bar{\rho}_{s} \boldsymbol{v}_{s}\right)=0 \\
\frac{\partial\left(\phi_{f} \bar{\rho}_{f}\right)}{\partial t}+\nabla\left(\phi_{f} \bar{\rho}_{f} \boldsymbol{v}_{s}\right)+\nabla \boldsymbol{n}_{f}=0
\end{gathered}
$$

where $\boldsymbol{v}_{s}$ corresponds to the absolute velocity of the solid phase and $\boldsymbol{n}_{f}$ is the water mass flux, described later. Note that no evaporation effect is considered in Eq. (15) since water evaporates only on the surface.

In the absence of external loads, the meat only shrinks depending on the moisture lost. $V(t)$ being the total volume of the product and $\phi_{f, 0}$ the initial volume fraction of the fluid, the following balance could be established:

$$
V(t)-V_{0}=\phi_{f} V(t)-\phi_{f, 0} V_{0}
$$

Since the solid matrix is always saturated with water, the product porosity coincides with the volumetric fraction of water $\phi_{f}$. For this reason, the porosity value can be calculated at each instant of time considering the solid skeleton of the product incompressible or quasi-incompressible $\left(J_{s} \approx 1\right)$ :

$$
\phi_{f}(t)=1-\frac{1-\phi_{f, 0}}{V(t) / V_{0}}=1-\frac{1-\phi_{f, 0}}{J(t)}
$$

In this way, the Jacobian associated with fluid or water loss $J(t)$ is a state function depending on the fluid content in the meat.

The heat transfer process inside the product, assuming the same temperature for all phases, can be modeled with a unique energy balance equation for the entire product. 


$$
\left(\rho C_{p}\right) \frac{\partial T}{\partial t}+\left(\boldsymbol{n}_{f} \cdot \nabla\left(C_{p, w} T\right)\right)=\nabla \cdot\left(k_{p} \nabla T\right)
$$

$C_{p}$ and $C_{p, w}$ are the specific heat of the product and the water, respectively, whereas $k_{p}$ is the thermal conductivity of the product.

Darcy's law, states that water flows in a porous medium due to the pressure gradient inside the solid matrix and gravity. Thus, the water mass flux can be written as:

$$
\boldsymbol{n}_{f}=-\rho_{f} \frac{\kappa}{\mu}\left(\nabla p_{f}-\rho_{f} \mathbf{g}\right)
$$

where $\kappa$ is the permeability of the medium and $\mu$ its dynamic viscosity. Considering the gravity effect insignificant and $p_{f}$ being the swelling pressure proportional to the difference between the actual $\rho_{f}$ and the equilibrium water concentration $\rho_{f, e q}(T)$ (Dhall and Datta, 2011):

$$
p_{f}=\vartheta\left(\rho_{f}-\rho_{f, e q}(T)\right)
$$

where $\vartheta$ is a constant of proportionality. Introducing this relation in (19):

$$
\boldsymbol{n}_{f}=-\left(D_{f} \nabla \rho_{f}-D_{f, T} \nabla T\right)
$$

$D_{f}=\rho_{f} \frac{\kappa}{\mu} \vartheta$ is the diffusivity due to the water gradient concentration and $D_{f, T}=\rho_{f} \frac{\kappa}{\mu} \vartheta \frac{\partial \rho_{f, e q}}{\partial T}$ is the diffusivity due to the temperature gradient, both taken as parameters determined through the model.

Once the swelling pressure is defined and the relevant simplifications are made, the evaporation front is limited to the surface of the material, so there 
is no internal steam generation. Therefore, the mass conservation equations are reduced only to that of liquid water:

$$
\frac{\partial \phi_{f} \bar{\rho}_{f}}{\partial t}+\nabla\left(\phi_{f} \bar{\rho}_{f} \boldsymbol{v}_{s}\right)=\nabla \cdot\left(D_{f} \nabla\left(\phi_{f} \bar{\rho}_{f}\right)+D_{f, T} \nabla T\right)
$$

\section{Materials and methods}

Longissimus dorsi muscles from two Asturiana de los Valles heifers (1 year old) 7 days post mortem were obtained the same day on which the experimental tests were performed. For the cooking tests, the middle parts of these loins were cut perpendicular to the longitudinal axis resulting into a total of eighteen steaks of three different thicknesses $(19 \pm 2 \mathrm{~mm}, 26 \pm 2$ $\mathrm{mm}$ and $34 \pm 2 \mathrm{~mm})$. From each steak, three pieces approximately $81 \pm 21$ $\mathrm{mm}$ long and $26 \pm 1 \mathrm{~mm}$ of wide were obtained cutting the steaks parallel to the grain. The weights of these pieces were $43.7 \pm 6.7 \mathrm{~g}, 51.9 \pm 12.9 \mathrm{~g}$ and $71.9 \pm 16.3 \mathrm{~g}$ for the thicknesses of 19, 26 and $34 \mathrm{~mm}$, respectively.

In order to determine the water holding capacity and to do the rheological measurements, the loin was sliced ( $4 \mathrm{~mm}$ thickness) and then cut into pieces of about $8 \mathrm{~g}$.

\subsection{Meat properties}

The density, the heat capacity and the heat conductivity of the solid phase of the meat were calculated as a function of temperature and composition (Choi and Okos, 1986). This composition was determined by the mass fractions of the protein $x_{\text {prot }}$ and fat $x_{\mathrm{fat}}$. The meat density was calculated as function of temperature and composition as follows (Nesvadba, 2014): 


$$
\bar{\rho}_{s}(T)=\left(\frac{x_{\text {prot }}}{\bar{\rho}_{\text {prot }}(T)}+\frac{x_{\text {fat }}}{\bar{\rho}_{\text {fat }}(T)}\right)^{-1}
$$

The specific heat of the meat $C_{p, s}(T)$ was defined for each component and then calculated using a mass fractions average mixing rule:

$$
C_{p, s}(T)=x_{\text {prot }} C_{p_{\text {prot }}}(T)+x_{\text {fat }} C_{p_{\text {fat }}}(T)
$$

Isotropic thermal conductivity is assumed for the product lying between two limiting values. The lower limit is given by a perpendicular model with all the constituents in layers perpendicular to the flow of heat $\frac{1}{k_{\perp}(T)}=\sum_{i} \frac{\phi_{i}}{k_{i}(T)}$. The upper limit is the parallel model, in which the constituents are arranged as parallel layers $k_{\|}(T)=\sum_{i} \phi_{i} k_{i}(T)$. The thermal conductivity of the product is estimated as:

$$
k_{p}(T)=g k_{\perp}(T)+(1-g) k_{\|}(T)
$$

where $g$ is a number between zero and one (Nesvadba, 2014; van der Sman, 2013).

\subsection{Water holding capacity}

Water holding capacity (WHC) describes the ability of the meat to resist the removal of liquid caused by protein denaturation during cooking. The WHC was measured following the procedure described by Goñi and Salvadori (2010). The effect of temperature on the WHC was determined by immersing slices of meat packaged into plastic bags in a thermostatic bath (Digiterm S-150, JP Selecta, Abrera, Spain) at a given temperature (from $30{ }^{\circ} \mathrm{C}$ to $100{ }^{\circ} \mathrm{C}$ ), and waiting for equilibrium (30 min until there was no more weight 
loss). The final water content in the meat was defined as the WHC. Ten replicas were used for each temperature and the results were expressed as $\mathrm{kg}$ water/kg dry material.

\subsection{Rheological measurement}

Rheological characteristics of circular beef samples with a thickness of $4 \pm 0.5 \mathrm{~mm}$ and a diameter of $50 \pm 2 \mathrm{~mm}$ were measured using a Physica MRC 301 rheometer (Anton Paar GmbH, Graz, Austria), equipped with serrated parallel plate geometry (50 mm, $4 \mathrm{~mm}$ gap) and a temperature controller $\left( \pm 0.5{ }^{\circ} \mathrm{C}\right)$. Dynamic oscillating analyses were performed at a frequency of $2 \mathrm{~Hz}$ and a constant stress of $3 \mathrm{~Pa}$. The constant value for frequency and stress were chosen within the linear viscoelastic region that was determined by performing frequency sweeps $(0.1-10 \mathrm{~Hz})$ and stress sweeps $(0.1-1000 \mathrm{~Pa})$. The tests were carried out increasing the sample temperature from $25{ }^{\circ} \mathrm{C}$ to $100{ }^{\circ} \mathrm{C}$ with steps of $5^{\circ} \mathrm{C}$, holding each temperature step for 3 min (enough to ensure no further changes in the measurement). The evolution of the storage modulus $\left(G^{\prime}\right)$ and phase angle, the ratio of loss modulus to storage modulus $(\phi)$, with temperature were recorded by the rheometer software using five replicas.

\subsection{Cooking procedure}

Each piece of meat (at $20^{\circ} \mathrm{C}$ ) was individually cooked on a multilayer 210 $\mathrm{mm}$ diameter, $5.5 \mathrm{~cm}$ deep round frying pan (WMF, WMF Group GmbH, Geislingen an der Steige, Germany). The bottom of the pan consisted of three layers: $0.6 \mathrm{~mm}$ of steel at the bottom, $3.5 \mathrm{~mm}$ of aluminium in the middle, and $0.8 \mathrm{~mm}$ of steel with a Teflon non stick coating at the top (Fig. 2.a). 
An induction hob (BOSCH Schott Ceran PXY675DW4E/01 model, BSH, Munich, Germany) was used for cooking (frying sensor at level 5). Once the hob is turned on, there is a transition period of approximately $110 \mathrm{~s}$ until a stable temperature at the pan surface is reached. When the thermographic images taken with an infrared thermal imager (875-2 model, Testo, Lenzkirch, Germany) indicated that a stable temperature of $215 \pm 3^{\circ} \mathrm{C}$ has been reached, the meat was added to the pan. From that moment on, the temperature drops slightly and recovers quickly, which makes it possible to consider that cooking takes place at a constant temperature at the pan surface of $215^{\circ} \mathrm{C}$. The meat was cooked at three degrees of doneness: very rare, medium rare and done, corresponding to cooking times of $180 \mathrm{~s}, 300 \mathrm{~s}$, and $420 \mathrm{~s}$, respectively, for the pieces of $19 \mathrm{~mm}$ thickness. For the pieces of 26 and $34 \mathrm{~mm}$ thickness, the cooking times were established by the model predictions. The samples were turned over at two thirds of the total cooking time. Six pieces obtained from steaks located in the loin at different longitudinal positions were cooked for each degree of doneness and thickness.

The meat weight was continuously measured by a balance placed under the induction hob (DS30K0.1L, Kern \& Sohn, Balinger-Frommern, Germany) with a precision of $0.1 \mathrm{~g}$. Data was recorded every 1s in a measurement range up to $30 \mathrm{~kg}$. The core temperature was measured by a penetration $T$ type, $1.5 \mathrm{~mm}$ diameter thermocouple connected to a data logger (177-T4, Testo, Lenzkirch, Germany), as shown in Fig. 2. The data were presented as the mean \pm standard deviation.

[Figure 2 about here.] 


\section{Finite element model}

A 3D computational model was developed to reproduce the cooking process. This model includes two different parts: an aluminium pan with a diameter of $210 \mathrm{~mm}$ and $5 \mathrm{~mm}$ thickness and a beef steak (see Fig. 3). The beef sample was modeled as a 3D rectangular cuboid object. To reproduce the turning over of the steak two pans were considered. First, the bottom face of the steak is heated in a pan. Secondly, the top face of the same steak is heated in a second pan. This method of simulating the turn over process is possible due to the insignificant relevance of gravity in this problem. In addition, in order to simplify the process, it has been considered that the steak surface remains flat during the entire cooking time.

The model recreates a quarter of this geometry. It was meshed with hexahedral elements using a quadratic approximation for mass transfer and temperature for the meat, and with tetrahedral elements for the pan. Mesh sensitivity analysis was carried out to establish the mesh size. The total number of degrees of freedom and elements is 5 and 366, respectively (300 elements for the beef sample).

[Figure 3 about here.]

The simulation time was fixed as the experimental cooking time for each degree of doneness. The boundary conditions on the top and bottom surfaces were reversed after turning over by activating the upper pan and deactivating the lower one. 


\subsection{Initial and boundary conditions}

An initial temperature condition of $215^{\circ} \mathrm{C}$ which remains constant throughout the cooking time is set on the surface of the pan to simulate the experimental procedure, as well as a uniform temperature for the meat of $20{ }^{\circ} \mathrm{C}$. The contact equation between the pan and the meat and the heat transfer general equation for the two faces are defined as:

$$
\begin{gathered}
-\left.k_{\text {pan }} \frac{\partial T}{\partial z}\right|_{z_{\text {pan }}=0}=-\left.k_{p} \frac{\partial T}{\partial z}\right|_{z_{\text {meat }}=0}=H_{c}\left(T_{\text {pan }}-T_{\text {surf }}\right) \\
q_{\text {surf }}=h\left(T_{\text {amb }}-T_{\text {surf }}\right)-\lambda n_{f, \text { surf }}-\mathbf{n}_{f} C_{p, w} T \cdot \mathbf{N}_{\text {surf }}
\end{gathered}
$$

where $T_{\text {pan }}, T_{\text {surf }}$ are the temperature of the pan and the temperature of the meat on the surface where the boundary condition is being evaluated, $k_{\text {pan }}$ is the conductivity of the pan and $\mathbf{N}_{\text {surf }}$ is the surface normal. The parameter $H_{c}$ refers to the thermal conductance between both surfaces. This parameter, which has been obtained computationally, regulates the heat flow received by the meat through the contact heating surface. Its value is crucial for this cooking method, so it has been one of the highlighted objectives of the study. $h$ is the thermal convection coefficient. $T_{a m b}$ is the temperature of the air surrounding the meat, $\lambda$ is the vaporization latent heat and $n_{f, s u r f}$ the magnitude of the evaporation flux. The meat around the heating surface increases its temperature very quickly so that the water holding capacity falls at a faster rate than water loss by evaporation, causing the dripping phenomenon (Hughes et al., 2014). Both, phase change to steam and the dripping of liquid water, were included as boundary condition on the surface of the meat in contact with the pan. On the surface in contact with the pan 
both, phase change to steam and the dripping of liquid water, were included, while on the side walls only the dripping was considered. Neither of these two phenomena occur on the upper face. This was reflected in the heat fluxes on the surfaces. The steam flow in the evaporation process is given in Eq. (28) and the drip losses in Eq. (29):

$$
\begin{gathered}
n_{f, \text { surf } E}=h_{m}\left(\rho_{v, \text { surf }}-\rho_{v, a m b}\right) \\
n_{f, \text { surf } D}=\mathbf{n}_{f} \cdot \mathbf{N}_{\text {surf }}-h_{m}\left(\rho_{v, \text { surf }}-\rho_{v, a m b}\right)
\end{gathered}
$$

where $h_{m}$ is the mass transfer coefficient by convection and $\rho_{v, \text { surf }}$ and $\rho_{v, a m b}$ are the vapor density on the surface of the meat and the vapor density in the surrounding air, respectively, obtained by the ideal gas law.

\subsection{Parameters}

The input parameters used in this model are shown in Table 1. These parameters were obtained through experimental tests measurements or from bibliography, while others were optimized through the model in order to fit the experimental results.

\section{Results and Discussion}

Firstly, the results of the meat parameters obtained by experimentation and necessary for the development of the model are explained. Validation of the model by comparing the central point temperature of the steak and the average moisture content is then shown. Lastly, the settings of the cooking 
Problem parameters

$T_{a m b}$ surrounding air temperature $\left[{ }^{\circ} \mathrm{C}\right]$

$T_{\text {pan }}$ pan temperature $\left[{ }^{\circ} \mathrm{C}\right]$

$P_{a m b}$ environment pressure $[\mathrm{kPa}]$

$H_{c}$ thermal conductance of pan-meat contact $\left[\mathrm{W} /\left(\mathrm{m}^{2} \mathrm{~K}\right)\right]$

$g$ thermal conductivity parameter

Water properties

$\bar{\rho}_{f}$ water density $\left[\mathrm{kg} / \mathrm{m}^{3}\right]$

$D_{f}$ water diffusivity $\left[\mathrm{m}^{2} / \mathrm{s}\right]$

$D_{f, T}$ water diffusivity due to temperature gradient $[\mathrm{kg} /(\mathrm{m} \mathrm{s} \mathrm{K})]$

$C_{p, f}$ water specific heat $\left[\mathrm{KJ} /\left(\mathrm{kg}{ }^{\circ} \mathrm{C}\right)\right]$

$k_{f}$ water thermal conductivity $[\mathrm{W} /(\mathrm{m} \mathrm{K})]$

$\lambda$ vaporization latent heat $[\mathrm{J} / \mathrm{kg}]$

$h_{m}$ mass transfer coefficient $[\mathrm{m} / \mathrm{s}]$

Meat properties

$C_{p, s}$ meat specific heat $[\mathrm{J} /(\mathrm{kg} \mathrm{K})]$

$k_{p}$ product thermal conductivity $[\mathrm{W} /(\mathrm{m} \mathrm{K})]$

WHC water holding capacity [ $\mathrm{kg}$ water $/ \mathrm{kg}$ dry material $]$

$G^{\prime}$ storage modulus $[\mathrm{kPa}]$
Value

Source

$\begin{array}{cc}25 & \text { Measured } \\ 215 & \text { Measured } \\ 1.013 \cdot 10^{2} & \text { Measured } \\ 120 & \text { Computational } \\ 0.45 & \text { Computational }\end{array}$

$\begin{array}{cc}997.2 & \text { Choi and Okos (1986) } \\ 1 \cdot 10^{-9} & \text { Computational } \\ D_{f} \cdot \frac{\partial \rho_{f, e q}}{\partial T} & \text { Computational } \\ 4.1289-9.0864 \cdot 10^{-5} \cdot T+5.4731 \cdot 10^{-6} \cdot T^{2} & \text { Choi and Okos }(1986) \\ 0.57 & \text { Choi and Okos (1986) } \\ 2.26 \cdot 10^{6} & \text { Straub (1985) } \\ 0.008 & \text { Computational }\end{array}$

$2.0082+1.2089 \cdot 10^{-3} \cdot T-1.3129 \cdot 10^{-6} \cdot T^{2} \quad$ Choi and Okos $(1986)$ $1.7881 \cdot 10^{-1}+1.1958 \cdot\left(10^{-3}\right) \cdot T-2.7178 \cdot\left(10^{-6}\right) \cdot T^{2} \quad$ Choi and Okos $(1986)$ Fig. $4 \quad$ Measured Fig. $5 \quad$ Measured

Table 1: Model input parameters.

times for the different thicknesses of meat based on the previous results are given.

\subsection{Effect of heating on meat properties}

\subsubsection{Water holding capacity}

The effect of temperature on the WHC is shown in Fig.4. As expected, the WHC diminishes as the temperature increases since the thermal denaturation of the proteins during cooking is the cause of the reduction of the water retention capacity of the meat. The evolution of WHC with temperature follows a sigmoidal shape, as previously described by Goñi and Salvadori (2010) and van der Sman (2007). The experimental values of WHC, determined using the Association of Official Analytical Chemists (AOAC) method no. 
950.46, are quite similar to those found by these authors for beef, although the values can vary from one type of muscle to another (Kondjoyan et al., 2013). The sarcomere length is known to have a deep effect on WHC. The mechanisms behind shortening of the sarcomere are complex and continue to be discussed (Ertbjerg and Puolanne, 2017). The following function was fitted to the experimental data:

$$
\operatorname{WHC}(T)=c_{i}-\frac{a_{1}}{1+a_{2} \exp \left(-a_{3}\left(T-T_{4}\right)\right)}
$$

where $c_{i}=2.986, a_{1}=1.69, a_{2}=0.56, a_{3}=0.08309$ and $T_{4}=66.76{ }^{\circ} \mathrm{C}$ were estimated by a non-linear regression using the Levenberg-Marquardt method and with a R-squared of 0.9853. In this way, the equilibrium water concentration is related to the WHC through the equation $\rho_{f, e q}(T)=$ $\operatorname{WHC}(T) \rho_{s}$.

[Figure 4 about here.]

\subsubsection{Rheological properties}

The temperature dependence of the storage modulus $G^{\prime}$ and the phase angle $\phi$ for the beef, as shown in Fig. 5, were obtained from the experimental tests described in section 3.3. The storage modulus decreases slightly until reaching a minimum value at $55{ }^{\circ} \mathrm{C}$. However, it increases markedly up to 40 $\mathrm{kPa}$ from $65{ }^{\circ} \mathrm{C}$ to $80{ }^{\circ} \mathrm{C}$, and decreases at temperatures above $80{ }^{\circ} \mathrm{C}$. The contraction of the connective tissue, which occurs mainly above $65{ }^{\circ} \mathrm{C}$, results in an increase in the elasticity of the meat, which leads to an increase in the storage modulus. The phase angle diminishes over the whole temperature range tested but more noticeable decrease is observed from $50{ }^{\circ} \mathrm{C}$ to $60{ }^{\circ} \mathrm{C}$ 
down to a plateau at around $65^{\circ} \mathrm{C}$. Tornberg (2005) and Rabeler and Feyissa (2018) obtained similar trends in the $30-80{ }^{\circ} \mathrm{C}$ range for $M$. biceps femoris beef and chicken breast, respectively, but the values found by these authors differ from those shown in Fig. 5 because they correspond to different muscles and species

[Figure 5 about here.]

The storage modulus, for temperatures between $30{ }^{\circ} \mathrm{C}$ and $100{ }^{\circ} \mathrm{C}$, can be defined by a piecewise Eq. (31) :

$$
G^{\prime}(T)= \begin{cases}G_{a} \cdot T+G_{b} & \text { if } \quad 30^{\circ} \mathrm{C} \leq \mathrm{T}<55^{\circ} \mathrm{C} \\ G_{c}+\frac{G_{d}}{\left(1+\exp \left(-G_{e}\left(T-G_{f}\right)\right)\right.} & \text { if } \quad 55^{\circ} \mathrm{C} \leq \mathrm{T}<80^{\circ} \mathrm{C} \\ G_{g} \cdot T^{2}+G_{h} \cdot T+G_{i} & \text { if } 80^{\circ} \mathrm{C} \leq T<100^{\circ} \mathrm{C}\end{cases}
$$

where $G_{a}=-0.8816 \mathrm{kPa}{ }^{\circ} \mathrm{C}^{-1}, G_{b}=82.06 \mathrm{kPa}, G_{c}=36.40 \mathrm{kPa}, G_{d}=$ $85.32 \mathrm{kPa}, G_{e}=0.3386, G_{f}=68.04, G_{g}=0.05647 \mathrm{kPa}^{\circ} \mathrm{C}^{-2}, G_{h}=-12.74$ $\mathrm{kPa}{ }^{\circ} \mathrm{C}^{-1}$ and $G_{i}=781.8 \mathrm{kPa}$, values obtained by adjusting the experimental results obtaining a R-squared of 0.9998 . Fig. 5 shows the experimental value of the storage modulus and its fitting.

\subsection{Temperature and water loss for pieces of $19 \mathrm{~mm}$}

Fig. 6 compares the temperature at the central point obtained with the model and the experimental results for the three degrees of doneness. In the experimental measuring, the sensor may suffer deviations in its position, 
which could produce differences in temperature measurements. These differences are reflected by the gray bands, which reflect the standard deviation of these measurements like in Figs. 6 to 9. Therefore, the accuracy of this initial thermocouple position was estimated as $\pm 1 \mathrm{~mm}$. For this reason, computational digressions were calculated considering this displacement of the location of the probe (see Fig. 6 d.). In every case a linear behavior was observed after $80 \mathrm{~s}$ of cooking. The maximum temperatures reached in the center of the steak for the different times were $33^{\circ} \mathrm{C}, 47^{\circ} \mathrm{C}$ and $58{ }^{\circ} \mathrm{C}$ for $180 \mathrm{~s}$ (very rare), $300 \mathrm{~s}$ (medium rare) and $420 \mathrm{~s}$ (done), respectively. The Root Mean Squared Error, RMSE, for each case was calculated as square root of the sum of the squared differences between the predicted and experimental values divided by the number of data. The RMSE obtained were: $2.16{ }^{\circ} \mathrm{C}$ (very rare), $3.56{ }^{\circ} \mathrm{C}$ (medium rare) and $4.57^{\circ} \mathrm{C}$ (done). These temperatures are very far from those obtained through the procedures described by the American Meat Science Association, AMSA (1995), for the different degrees of meat doneness, $\left(55^{\circ} \mathrm{C}\right.$-very rare, $60{ }^{\circ} \mathrm{C}$-rare, $63{ }^{\circ} \mathrm{C}$-medium rare, $71{ }^{\circ} \mathrm{C}$-medium, $7{ }^{\circ} \mathrm{C}$-done and $82{ }^{\circ} \mathrm{C}$-well done). Leaving aside microbiological considerations, from the point of view of consumer acceptance it is very difficult to establish a relationship between the internal cooking temperature and the perception of the degree of cooking which depends on consumer preferences (López Osornio et al., 2008). Fig. 6.d shows the temperature distribution in a cross section for different times of medium rare cooking degree. The temperature of the face in contact with the pan rises quickly and stabilizes after a few seconds. Contrary to the temperature of the meat core, this temperature is much higher $\left(120^{\circ} \mathrm{C}\right)$. After turning the steak over, a 
reduction in this temperature is observed as this face is no longer in contact with the heat source. It is now the other face which suffers an increase in temperature. The cooking process was stopped the moment at which the difference in temperature between the central point inside the steak and the central point on the surface in contact with the pan was similar to the difference of temperature at the moment of turning over the steak $\left(97^{\circ} \mathrm{C}\right.$ - very rare, $93{ }^{\circ} \mathrm{C}$ - medium rare, $87^{\circ} \mathrm{C}$ - done). Therefore, the time of turning over the steak coincides with two thirds of the total cooking time.

As regards shrinkage, Fig. 6.d shows the volume reduction of the steak and its change of shape for the medium rare degree of cooking. At the beginning, the greatest deformation appears near the pan, while in the central part of the steak there is hardly any deformation as this is the coldest area of the piece of meat. As time progresses, this deformation extends to the central part. At the moment of turning over the steak, the maximum deformation occurs again in the face in contact with the pan. At $40 \mathrm{~s}$ the volume reduction is around $1 \%$ while at the end of the cooking (300 s) it is around $9 \%$. In the case of the done degree of cooking (420 s), the final volume reduction is about $12 \%$. The evolution of shrinkage during cooking is a consequence of an increasing rigidity of the myofibrillar structure due to the thermal denaturation of proteins. At temperatures from $40{ }^{\circ} \mathrm{C}$ to $60{ }^{\circ} \mathrm{C}$ transverse shrinkage occurs in the miofibrils attributed principally to myosin, and in the temperature range from $70{ }^{\circ} \mathrm{C}$ to $80{ }^{\circ} \mathrm{C}$ it is longitudinal and attributed fundamentally to actin (Hughes et al., 2014; Purslow et al., 2016). The juice expelled by the protein denaturation and contraction is associated with the water loss during cooking that occurs from $45{ }^{\circ} \mathrm{C}$ to $75{ }^{\circ} \mathrm{C}-80{ }^{\circ} \mathrm{C}$, and 
above $80{ }^{\circ} \mathrm{C}$ the cooking loss diminishes gradually (Tornberg, 2005). The water loss evolution of the meat for the different cooking degrees is shown in Fig. 7. The cooking losses for the different cooking times were $4 \%, 7$ $\%$ and $10 \%$ for $180 \mathrm{~s}$ (very rare), $300 \mathrm{~s}$ (medium rare) and $420 \mathrm{~s}$ (done), respectively. The computational results fit optimally with experimentation, demonstrating a linear behavior with time. The RMSE for each case in our study is: $1.48 \%$ (very rare), $2.08 \%$ (medium rare) and $2.40 \%$ (done). The above-mentioned research of Dhall and Datta (2011) shows that a water loss of $7 \%$ can be obtained for a cooking time of $300 \mathrm{~s}$ in patties, quite similar to our medium rare degree of cooking. It is not surprising that similar results are obtained between minced meat and whole meat since there is evidence of the minor role of collagen in the loss of water during cooking (Hughes et al., 2014; Tornberg, 2005).

[Figure 6 about here.]

[Figure 7 about here.]

\subsection{Cooking times prediction for different thicknesses of meat: water loss as indicator}

One of the possible applications of the modeling of pan cooking is to provide assistance during cooking, and so it is important to know how the model can be adapted to different real cooking conditions. One of the parameters that can most influence meat cooking results is the thickness of the steak. In order to predict how the cooking time changes depending on the thickness of the meat, weight loss has been established as a control variable. In the same manner as with the $19 \mathrm{~mm}$ thickness, experimental cooking tests were 
carried out with thicknesses of $26 \mathrm{~mm}$ and $34 \mathrm{~mm}$. The time of turning over the steak and the final time of cooking were fixed at the moment when the meat reached the same water loss than as the $19 \mathrm{~mm}$ piece in each degree of cooking. Thus, the times for the $26 \mathrm{~mm}$ thickness were modified to $225 \mathrm{~s}$ (very rare), $450 \mathrm{~s}$ (medium rare) and $720 \mathrm{~s}$ (done), and the times for $34 \mathrm{~mm}$ were $265 \mathrm{~s}$ (very rare), $495 \mathrm{~s}$ (medium rare) and $770 \mathrm{~s}$ (done). The times for turning over the steak were kept at two thirds of the total time. Once the model for the $19 \mathrm{~mm}$ thickness had been verified, it was checked whether this model could adjust the temperature in the center of the steak and the water loss for these new thicknesses. These results are shown in Fig. 8 and in Fig. 9. The model successfully adjusts both the 26 and $34 \mathrm{~mm}$ cases. In the same way as for the $19 \mathrm{~mm}$ pieces, a displacement deviation of $5 \%$ in the location of the temperature probe was applied in each case. The maximum temperatures reached in the center of the steak for each thickness and time are quite similar to those obtained with the $19 \mathrm{~mm}$ thickness steak: $26 \mathrm{~mm}$ $\left(31{ }^{\circ} \mathrm{C}\right.$ - very rare, $45{ }^{\circ} \mathrm{C}$ - medium rare, $58{ }^{\circ} \mathrm{C}$ - done $)$ and $34 \mathrm{~mm}\left(32{ }^{\circ} \mathrm{C}\right.$ - very rare, $46{ }^{\circ} \mathrm{C}$ - medium rare, $58{ }^{\circ} \mathrm{C}$ - done). This verification could be taken as evidence of an appropriate functioning of the model. As a conclusion of these results, we can confirm that this model may predict cooking times according to the weight loss of the meat during cooking.

[Figure 8 about here.]

[Figure 9 about here.] 


\subsection{Analyzing the effect of the steak thickness}

The thickness of the fillets is not uniform and some uncertainties are present in the measurement process. Small variations in the thickness of the meat could cause changes in the expected temperature reached in the steak. Changes in temperature in the presence of variations in thickness can be known by means of a sensitivity analysis carried out with the Monte Carlo method. This technique has been applied to the 3D model of the steak of $19 \mathrm{~mm}$ of thickness considering the three cooking degrees. The results are shown in Fig. 10 for the done degree where a population of one hundred models was analyzed considering a uniform distributed thickness between $\pm 10 \%$ the mean value. In order to reduce the computational cost, the model was simplified by disabling the effect of shrinkage, hence the small differences in temperature and weight loss compared with those analyzed in section 5.2 for thicknesses of $19 \mathrm{~mm}$. The temperature at the central point is represented as a mean value and a standard deviation (Fig. 10.a) and takes values of $35 \pm 2$ ${ }^{\circ} \mathrm{C}, 47 \pm 4{ }^{\circ} \mathrm{C}$ and $63 \pm 5{ }^{\circ} \mathrm{C}$ at $180 \mathrm{~s}$ (very rare), $300 \mathrm{~s}$ (medium rare) and 420 $\mathrm{s}$ (done), respectively. A sensitivity analysis was also conducted on the meat water loss (Fig. 10.b) obtaining values of $4 \pm 0.3 \%, 7 \pm 0.4 \%$ and $10 \pm 0.5$ $\%$ at $180 \mathrm{~s}$ (very rare), $300 \mathrm{~s}$ (medium rare) and $420 \mathrm{~s}$ (done), respectively. These dispersion values obtained with the Monte Carlo technique are very close to those obtained in the experimental tests.

[Figure 10 about here.] 


\section{Conclusions}

A 3D computational model was developed that considers the phenomena of heat and moisture flow transfer and the deformation of meat during the double-sided pan cooking of beef steaks. The equations were solved by the finite element method. The evolution over time of the temperature at the central point and the weight loss predicted by the model were compared with the experimental results for different cooking times and meat thicknesses. The good agreement between the predicted and experimental results allowed the model to be verified and the assumptions made to be considered appropriate.

The simulation results provided a better and more detailed insight into steak pan cooking allowing the accurate prediction of the cooking time required to reach a certain temperature in the center of the meat, that is, to achieve the desired degree of doneness regardless of the steak thickness; this being of utmost importance for successful cooking. The choice of weight loss as the reference parameter to estimate the cooking times of steaks of different thicknesses is a promising option for several reasons: the core temperatures thus obtained for the different thickness are similar $\left( \pm 2{ }^{\circ} \mathrm{C}\right)$, the measurement of the weight may be implemented in induction hobs in the future, the difficulty of measuring the temperature exactly at the geometric center of the steak is overcome, and a small deviation in fillet thickness involves a change in temperature prediction at the center of the same order of magnitude as that between some degrees of doneness. However, since the water retention capacity depends on the muscle and the quality of the meat, the use of weight loss as the only reference parameter to establish the cooking 
time has its limitations for uncharacterized pieces of meat, but it can still be considered a complementary parameter to the central temperature.

\section{Acknowledgements}

This work has been funded by the Spanish Ministry of Science, Innovation and Universities through the RETOS-COLABORATION 2017 program (project RTC-2017-5965-6, ARQUE), co-financed by the European Union with ERDF; and by the BSH Home Appliances Group. It has also been supported by the Department of Industry and Innovation (Government of Aragon) through the research group Grant T24-20R and T07-20R (cofinanced by Feder 2014-2020: Construyendo Europa desde Aragon). J. Moya was supported by the Government of Spain, order CNU/692/2019.

\section{Declaration of Interest Statement}

The authors have no competing interests to declare

\section{References}

A.K. Datta. Porous media approaches to studying simultaneous heat and mass transfer in food processes. i: Problem formulations. Journal of Food Engineering, 80(1):80-95, may 2007. doi: 10.1016/j.jfoodeng.2006.05.013.

R.G.M. van der Sman. Biopolymer gel swelling analysed with scaling laws and flory-rehner theory. Food Hydrocolloids, 48:94-101, jun 2015. doi: 10.1016/j.foodhyd.2015.01.025. 
A. H. Feyissa, K. V. Gernaey, and J. Adler-Nissen. 3d modelling of coupled mass and heat transfer of a convection-oven roasting process. Meat Science, 93(4):810-820, apr 2013. doi: 10.1016/j.meatsci.2012.12.003.

S. Ahmad, M. Ali Khan, and M. Kamil. Mathematical modeling of meat cylinder cooking. LWT - Food Science and Technology, 60(2):678-683, mar 2015. doi: 10.1016/j.lwt.2014.10.061.

H. Nelson, S. Deyo, S. Granzier-Nakajima, P. Puente, K. Tully, and J. Webb. A mathematical model for meat cooking. The European Physical Journal Plus, 135(3), mar 2020. doi: 10.1140/epjp/s13360-020-00311-0.

M. J. Blikra, D. Skipnes, and A. H. Feyissa. Model for heat and mass transport during cooking of cod loin in a convection oven. Food Control, 102:29-37, aug 2019. doi: 10.1016/j.foodcont.2019.03.001.

A.K. Datta. Toward computer-aided food engineering: Mechanistic frameworks for evolution of product, quality and safety during processing. Journal of Food Engineering, 176:9-27, may 2016. doi: 10.1016/j.jfoodeng.2015.10.010.

E. Tornberg. Effects of heat on meat proteins - implications on structure and quality of meat products. Meat Sci., 70(3):493-508, 2005. doi: 10.1016/j.meatsci.2004.11.021.

E. Tornberg. Engineering processes in meat products and how they influence their biophysical properties. Meat Science, 95(4):871-878, dec 2013. doi: 10.1016/j.meatsci.2013.04.053. 
F. Rabeler and A. H. Feyissa. Modelling the transport phenomena and texture changes of chicken breast meat during the roasting in a convective oven. Journal of Food Engineering, 237:60-68, nov 2018. doi: 10.1016/j.jfoodeng.2018.05.021.

H. S. Bansal, P. S. Takhar, C. Z. Alvarado, and L. D. Thompson. Transport mechanisms and quality changes during frying of chicken nuggetshybrid mixture theory based modeling and experimental verification. Journal of Food Science, 80(12):E2759-E2773, oct 2015. doi: 10.1111/17503841.13082 .

H. Isleroglu and F. Kaymak-Ertekin. Modelling of heat and mass transfer during cooking in steam-assisted hybrid oven. Journal of Food Engineering, 181:50-58, jul 2016. doi: 10.1016/j.jfoodeng.2016.02.027.

A. Kondjoyan, S. Oillic, S. Portanguen, and J. B. Gros. Combined heat transfer and kinetic models to predict cooking loss during heat treatment of beef meat. Meat Science, 95(2):336-344, oct 2013. doi: 10.1016/j.meatsci.2013.04.061.

R.G.M. van der Sman. Moisture transport during cooking of meat: An analysis based on flory-rehner theory. Meat Science, 76(4):730-738, aug 2007. doi: 10.1016/j.meatsci.2007.02.014.

K.N. van Koerten, D. Somsen, R.M. Boom, and M.A.I. Schutyser. Modelling water evaporation during frying with an evaporation dependent heat transfer coefficient. Journal of Food Engineering, 197:60-67, mar 2017. doi: 10.1016/j.jfoodeng.2016.11.007. 
A. Dhall and A. K. Datta. Transport in deformable food materials: A poromechanics approach. Chemical Engineering Science, 66(24):64826497, dec 2011. doi: 10.1016/j.ces.2011.09.001.

J. F. Eberth, J. A. Neal, and F. C. Robles Hernandez. Evaluation of heat propagation through poultry in a reduced computational-cost model of contact cooking. International Journal of Food Science \& Technology, 47 (6):1130-1137, mar 2012. doi: 10.1111/j.1365-2621.2012.02951.x.

R. Rocca-Poliméni, N. Z. Vilet, S. Roux, J. L. Bailleul, and B. Broyart. Continuous measurement of contact heat flux during minced meat grilling. Journal of Food Engineering, 242:163-171, feb 2019. doi: 10.1016/j.jfoodeng.2018.08.032.

N. Myhrvold. Modernist cuisine: el arte y la ciencia de la cocina. Taschen Benedikt, 2017.

P. J. Flory. Phase changes in proteins and polypeptides. Journal of Polymer Science, 49(151):105-128, jan 1961. doi: 10.1002/pol.1961.1204915106.

L. Vujosevic and V. A. Lubarda. Finite-strain thermoelasticity based on multiplicative decomposition of deformation gradient. Theoretical and applied mechanics, 28(29):379-399, 2002. doi: 10.2298/TAM0229379V.

Y. Choi and M. R. Okos. Effects of temperature and composition on thermal properties of foods. Journal of Food Process and Applications, 1(1):93-101, 1986. 
P. Nesvadba. Thermal properties of unfrozen foods. In Engineering Properties of Foods, Fourth Edition, pages 223-246. CRC Press, apr 2014. doi: 10.1201/b16897-8.

R.G.M. van der Sman. Modeling cooking of chicken meat in industrial tunnel ovens with the flory-rehner theory. Meat Science, 95(4):940-957, dec 2013. doi: 10.1016/j.meatsci.2013.03.027.

S. M. Goñi and V. O. Salvadori. Kinetic modelling of colour changes during beef roasting. Procedia Food Science, 1:1039-1044, 2010. doi: 10.1016/j.profoo.2011.09.155.

J.M. Hughes, S.K. Oiseth, P.P. Purslow, and R.D. Warner. A structural approach to understanding the interactions between colour, water-holding capacity and tenderness. Meat Science, 98(3):520-532, nov 2014. doi: 10.1016/j.meatsci.2014.05.022.

J. Straub. NBS/NRC steam tables. VonL. haar, j. s. gallagher undG. s. kell. hemisphere publishing corp., washington-new york-london 1984. 1. aufl., XII, 320 s., geb., \$ 34.50. Chemie Ingenieur Technik, 57(9):812-812, 1985. doi: $10.1002 /$ cite.330570931.

P. Ertbjerg and E. Puolanne. Muscle structure, sarcomere length and influences on meat quality: A review. Meat Science, 132:139-152, oct 2017. doi: 10.1016/j.meatsci.2017.04.261.

M.M. López Osornio, G. Hough, A. Salvador, E. Chambers, S. McGraw, and S. Fiszman. Beef's optimum internal cooking temperature as seen by consumers from different countries using survival analysis 
632

633

statistics. Food Quality and Preference, 19(1):12-20, jan 2008. doi: 10.1016/j.foodqual.2007.06.004.

P.P. Purslow, S. Oiseth, J. Hughes, and R.D. Warner. The structural basis of cooking loss in beef: Variations with temperature and ageing. Food Research International, 89:739-748, nov 2016. doi: 10.1016/j.foodres.2016.09.010. 


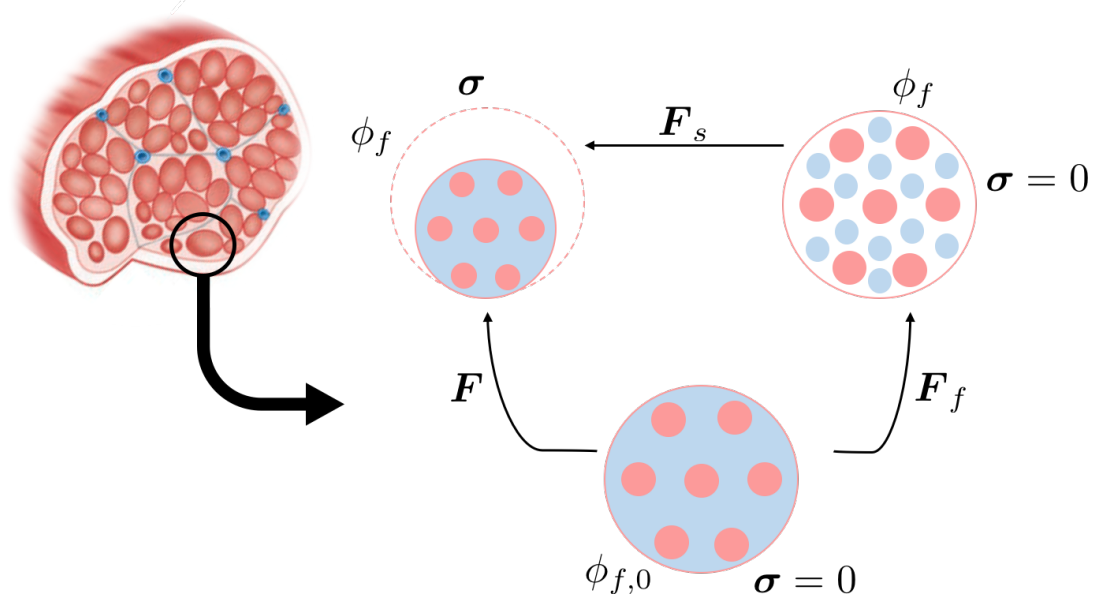

Figure 1: Modeling the moisture loss and deformation of the meat using a fictitious intermediate step. Quasi-static equilibrium was considered $(\nabla \boldsymbol{\sigma}=0$ with $\boldsymbol{\sigma}$ being the Cauchy stress tensor) and the deformation gradient tensor $\boldsymbol{F}$ was decomposed multiplicatively in two parts associated with the water volume change, $\boldsymbol{F}_{f}$, and the elastic deformation of the solid phase $\boldsymbol{F}_{s}$. 


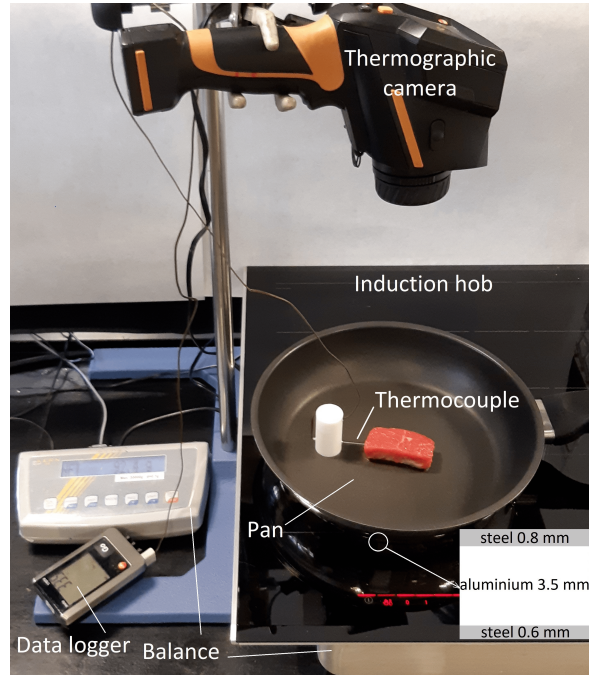

(a)

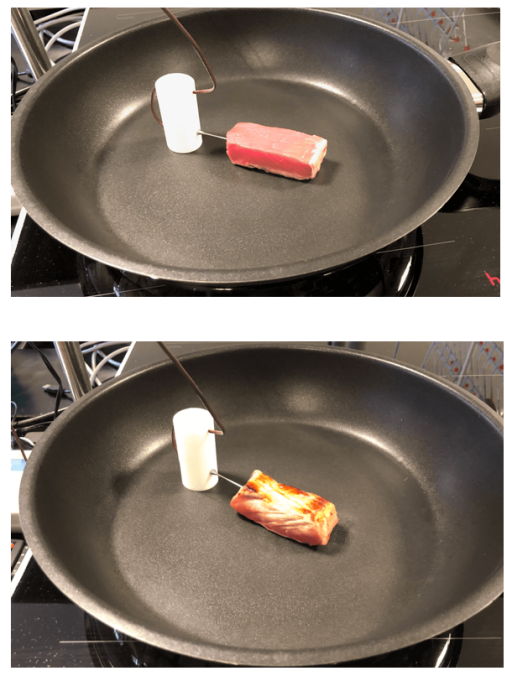

(b)

Figure 2: a) Experimental setup for temperature and weight loss measurement during the cooking process. b) Evolution of the geometry of the steak along the test comparing the beginning of the process (top) and the end (bottom) for a done doneness degree. 


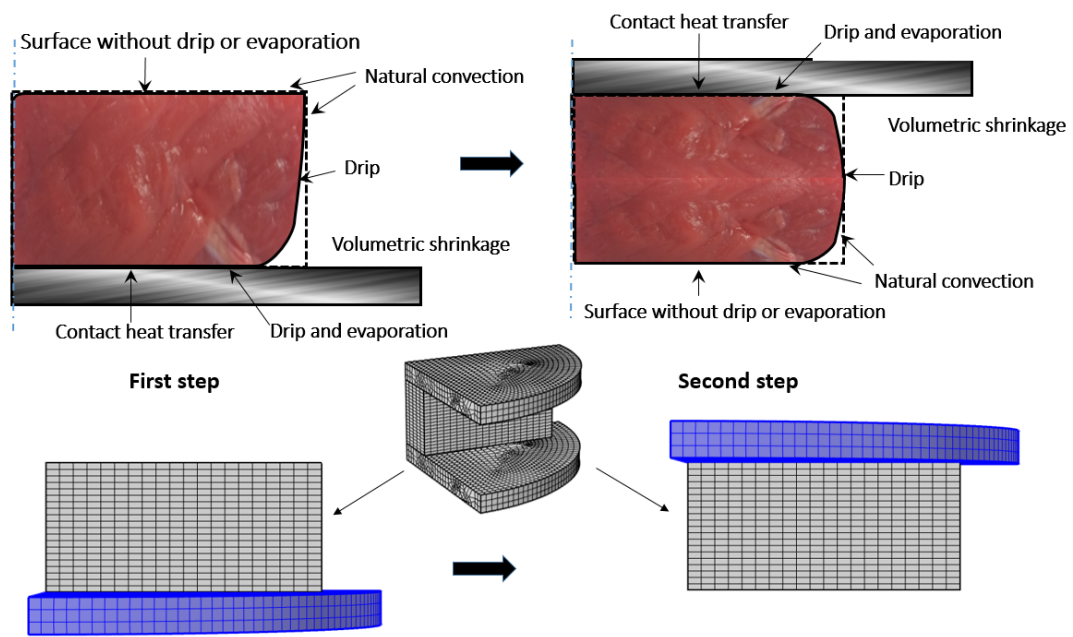

Figure 3: Finite element model and strategy defined for the turned over meat. 


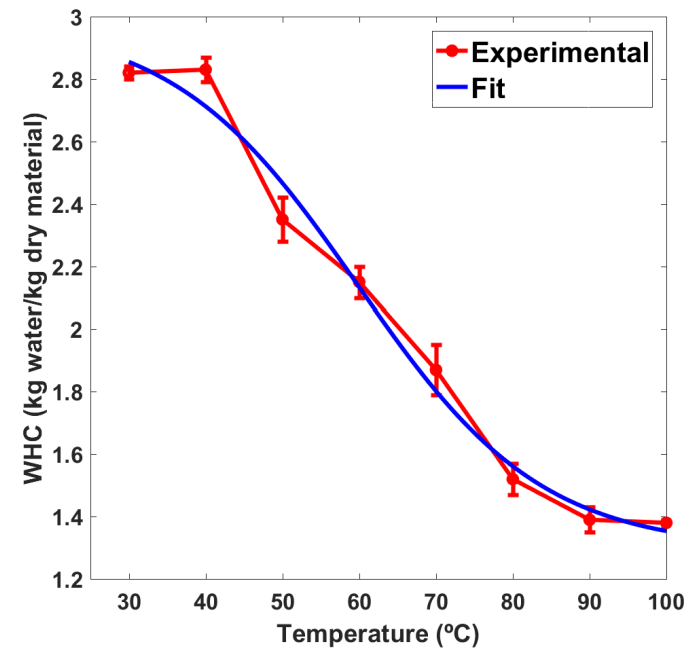

Figure 4: Water Holding Capacity as a function of temperature $T$ for beef meat. 


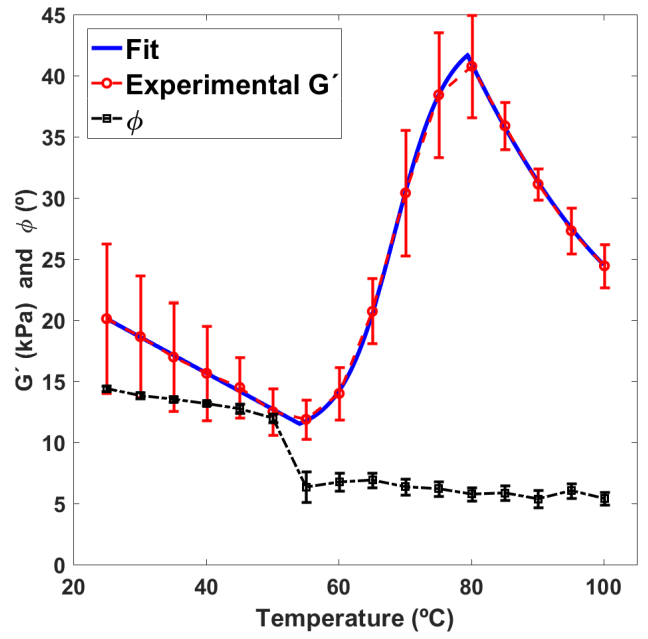

Figure 5: Storage modulus, $G^{\prime}(\mathrm{kPa})$, and phase angle, $\phi\left(^{\circ}\right)$, for beef $M$. Longissimus dorsi as a function of cooking temperature. Experimental values indicated by symbols and estimated values by the blue line. 


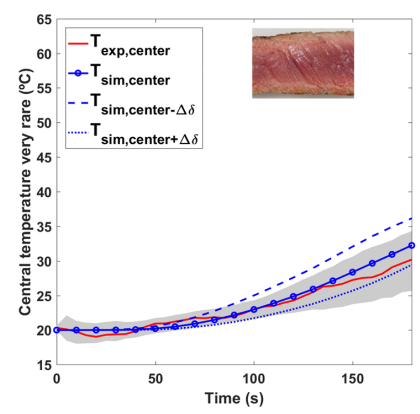

(a)

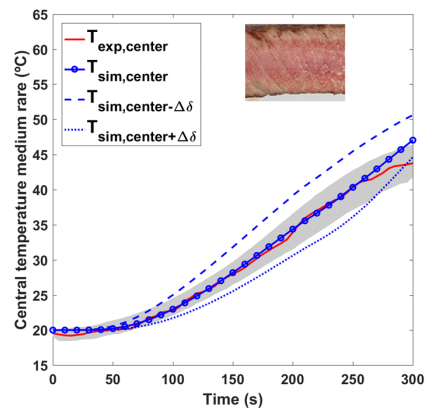

(b)

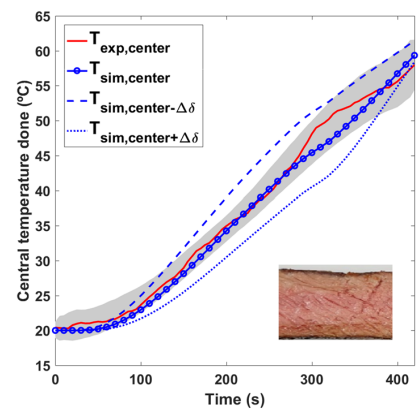

(c)

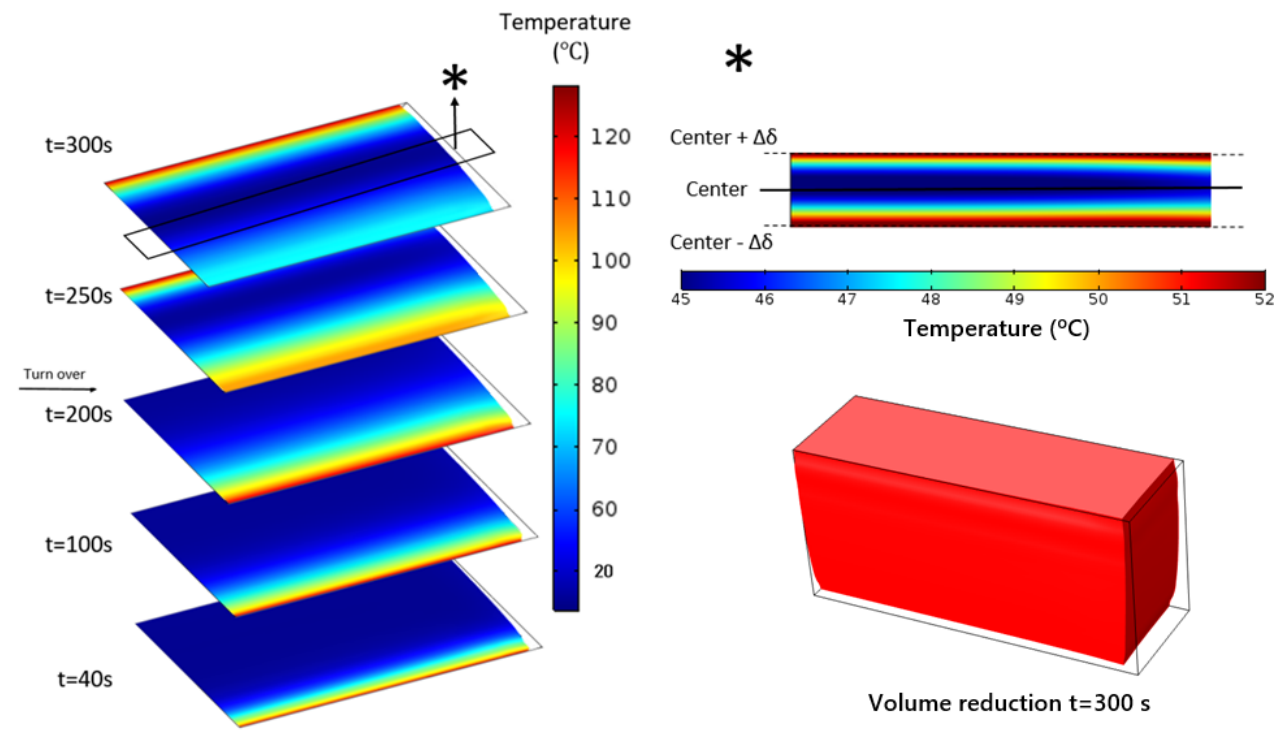

(d)

Figure 6: Central temperature evolution for $19 \mathrm{~mm}$ of thickness: a) very rare, b) medium rare, c) done cooking degree. d) Temperature distribution in a cross section for different times in case of medium rare cooking degree, central section temperature gradient considering the sensor located at $\Delta \delta= \pm 1 \mathrm{~mm}$, and volume reduction. 


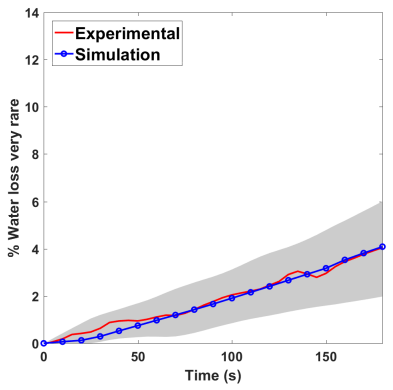

(a)

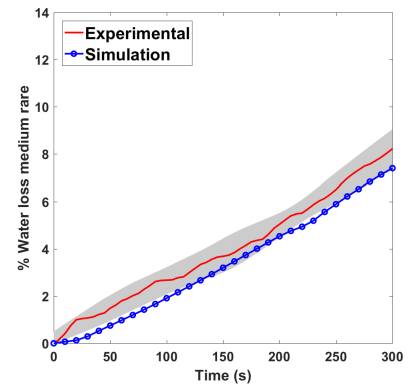

(b)

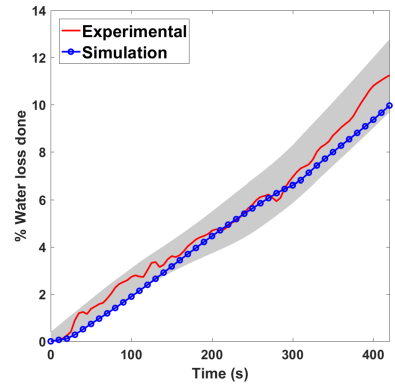

(c)

Figure 7: Water loss evolution for $19 \mathrm{~mm}$ of thickness: a) very rare, b) medium rare, c) done cooking degrees. 


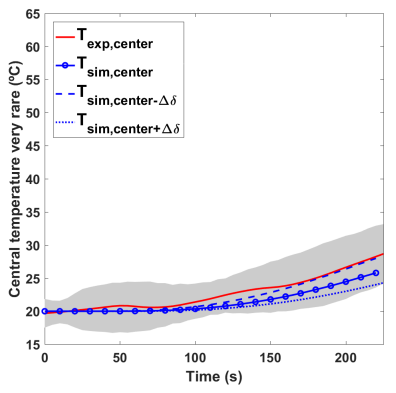

(a)

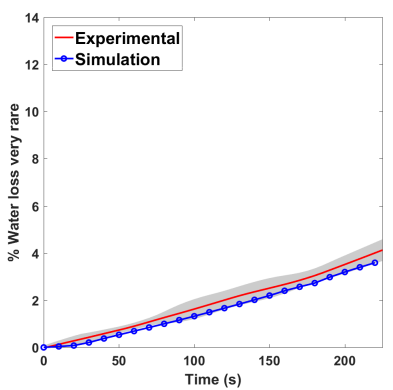

(d)

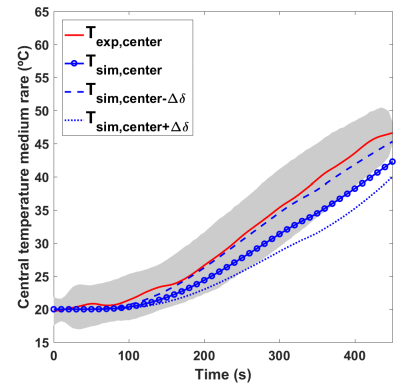

(b)

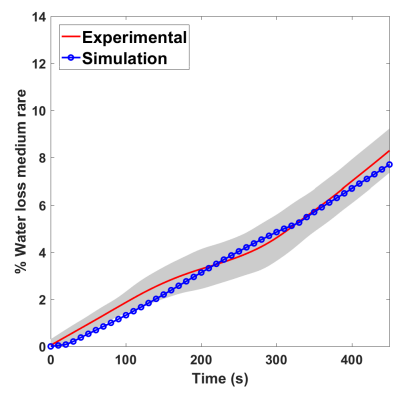

(e)

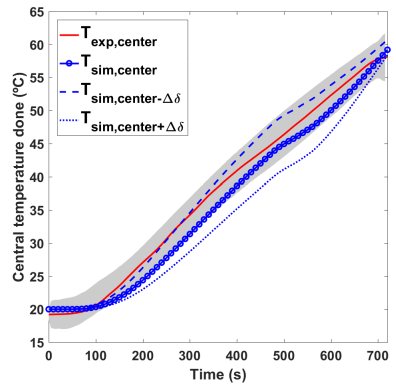

(c)

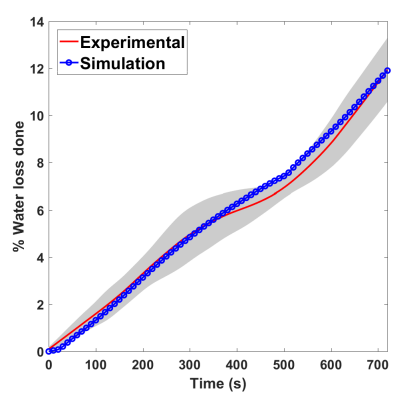

(f)

Figure 8: Central temperature evolution for $26 \mathrm{~mm}$ thickness: a) very rare, b) medium rare, c) done cooking degrees. Water loss evolution for d) very rare, e) medium rare, f) done. 


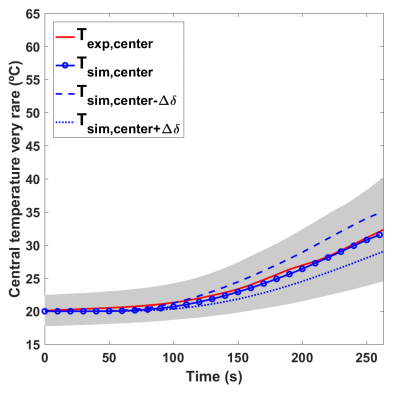

(a)

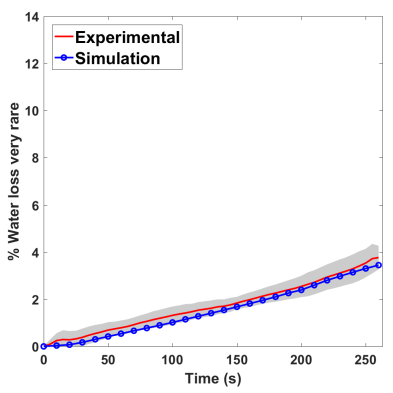

(d)

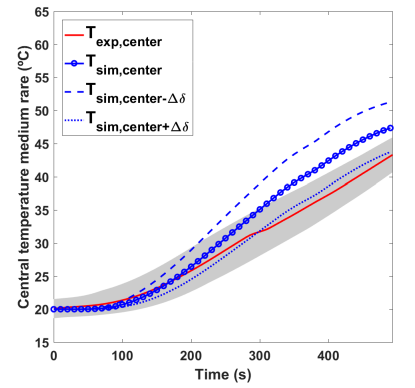

(b)

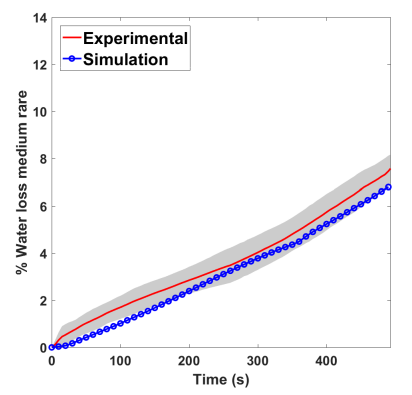

(e)

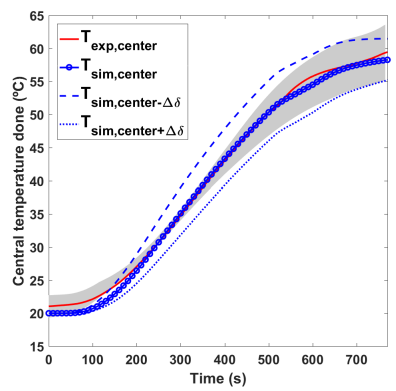

(c)

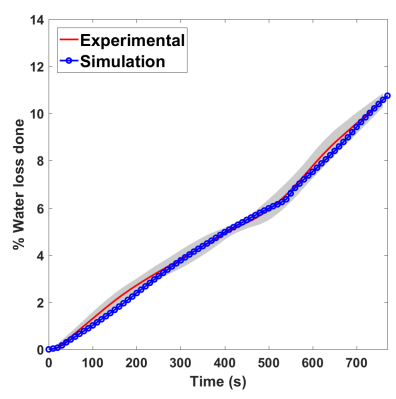

(f)

Figure 9: Central temperature evolution for $34 \mathrm{~mm}$ thickness: a) very rare, b) medium rare, c) done cooking degrees. Water loss evolution for d) very rare, e) medium rare, f) done. 


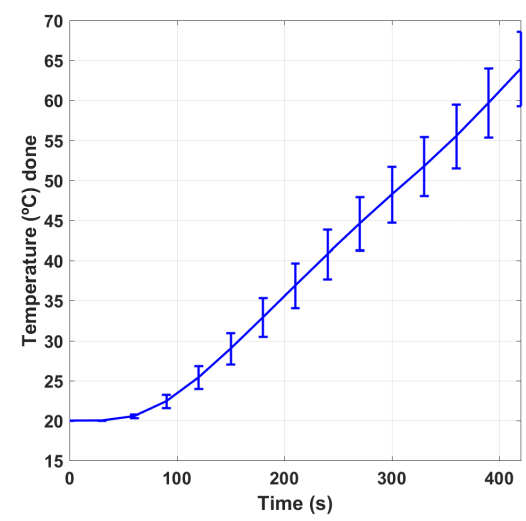

(a)

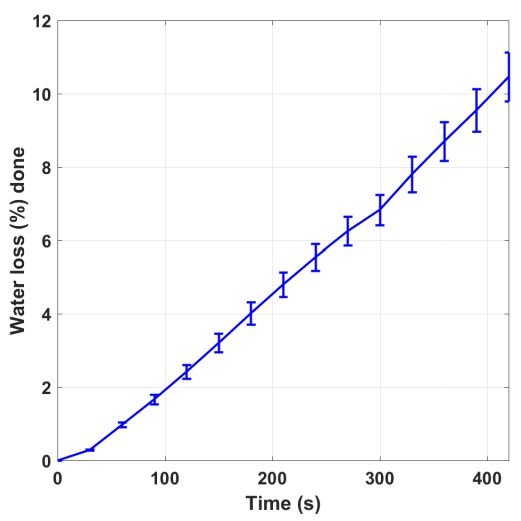

(b)

Figure 10: Sensitivity analysis of a) central temperature and b) water loss regarding variations in thickness for the done degree. 